\title{
The principal determinants for the structure of the substrate-binding pocket are located within a central core of a biphenyl dioxygenase a subunit
}

\author{
Marco Zielinski, Silke Backhaus and Bernd Hofer
}

\begin{abstract}
Author for correspondence: Bernd Hofer. Tel: +49 531 6181467. Fax: +49 5316181411. e-mail: bho@gbf.de
\end{abstract}

German Research Centre for Biotechnology (GBF), Division of Microbiology, Mascheroder Weg 1, D-38124 Braunschweig, Germany
Protein engineering by segment exchange was used to distinguish between regions of major and minor influence on the structure of the substrate-binding pocket of a biphenyl dioxygenase (BDO). Eight chimaeric enzyme systems were generated that each consisted of a hybrid hydroxylase $\alpha$ subunit (BphA1) containing segments from Burkholderia sp. strain LB400 and Rhodococcus globerulus P6, and of a hydroxylase $\beta$ subunit (BphA2), a ferredoxin (BphA3) and a ferredoxin reductase (BphA4) from strain LB400. All hybrid bphA1 genes were expressed at high levels. Seven of the resulting fusion subunits functionally interacted with the other polypeptides of the dioxygenase system to yield catalytically active enzymes. Changes in the regiospecificity of substrate attack, monitored by the formation of seventeen different dioxygenation products obtained from seven chlorobiphenyls, were used to monitor effects of segment exchanges on the structure of the BDO substratebinding site. Exchanges of neither the $\beta$ subunit nor the $\mathrm{N}$ - and C-terminal regions of the $\alpha$ subunit exerted significant influences. All BDO regions that showed major effects on the substrate-binding pocket were located between approximately positions 165 and 395 of the $\alpha$ subunit. Within this part of the enzyme, in addition to segments identified previously, a subregion which is involved in ligation of the mononuclear iron significantly influenced the regiospecificity of substrate dioxygenation. Moreover, the results indicate that the construction of appropriate hybrid genes may be used as a general strategy to overcome problems in obtaining heterologous BDO activities in Escherichia coli or other host organisms.

Keywords: aerobic PCB catabolism, biphenyl dioxygenase, chlorobiphenyls, heterologous gene expression, substrate-binding site

\section{INTRODUCTION}

The prototype reaction catalysed by aromatic-ringhydroxylating dioxygenases (ARHDOs) is the addition of two hydroxyl groups to vicinal carbons, thereby destroying the aromatic system and yielding dihydrodiol compounds of cis, cis stereochemistry (Fig. 1) (Butler \& Mason, 1997; Boyd \& Sheldrake, 1998). This family of enzymes is of increasing interest for different applications. One is the stereospecific synthesis of chiral

Abbreviations: ARHDO, aromatic-ring-hydroxylating dioxygenase; BDO, biphenyl dioxygenase; $C B$, chlorobiphenyl; $O E$, overlap extension; $P C B$, polychlorobiphenyl. synthons (Boyd \& Sheldrake, 1998; Hudlicky et al., 1999), another is the degradation of aromatic pollutants (Butler \& Mason, 1997; Gibson \& Parales, 2000). ARHDOs are key enzymes in the aerobic microbial metabolism of such compounds.

Our current interest focuses on the use of ARHDOs as catalysts for the removal of polychlorobiphenyls (PCBs). A particular problem lies in the fact that technical PCBs are mixtures of dozens of compounds, so-called congeners. A strategy to overcome this difficulty is the generation of enzymes with novel substrate specificities by protein engineering. Sequence elements of biphenyl dioxygenases (BDOs) that significantly influence substrate specificity have been identified by exchanges of 


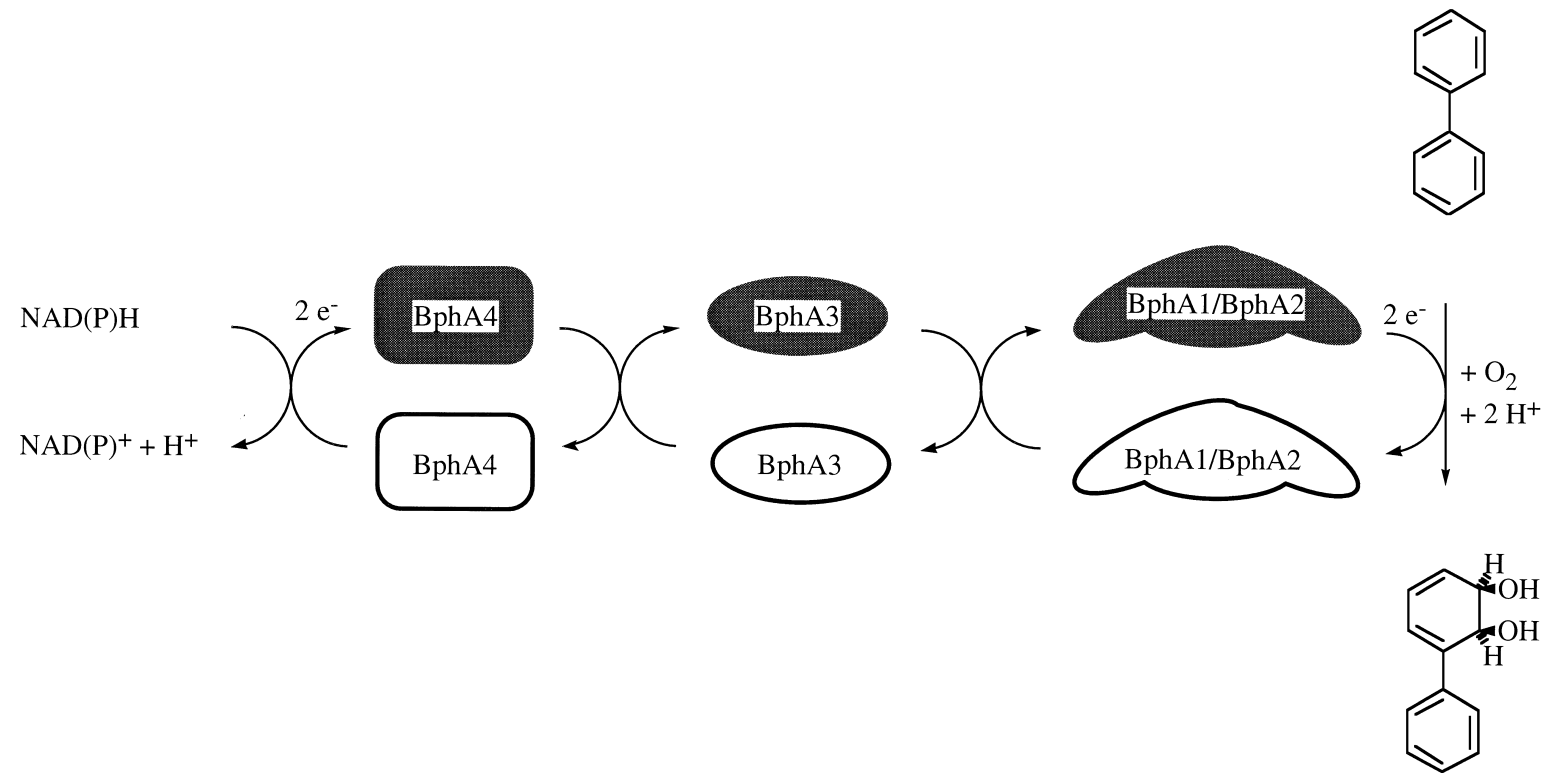

Fig. 1. Reaction catalysed by ARHDOs and functions of the polypeptides of class II and class III ARHDO systems, exemplified with a BDO. BphA1 ( $\alpha$ subunit)/BphA2 ( $\beta$ subunit) complex is the hydroxylase; BphA3 is ferredoxin; BphA4 is ferredoxin reductase. Reduced and oxidized forms of proteins are indicated by shaded and white symbols, respectively.

entire subunits or parts of $\alpha$ subunits (Erickson \& Mondello, 1993; Furukawa et al., 1994; Hirose et al., 1994; Kimura et al., 1997; Mondello et al., 1997). These approaches used segments of the $b p h$-encoded large subunits of Burkholderia sp. strain LB400 and Pseudomonas pseudoalcaligenes KF707 which possess different substrate spectra and differ by only 20 aa. Whilst this small number of differences permits a fast identification of regions of importance, the influence of segments with no or few differences cannot reliably be assessed by this approach. There is no a priori reason to exclude the possibility that such regions can crucially influence substrate binding. Rather, our general knowledge on enzyme structure and function suggests that more than the relatively small number of amino acids identified in the above-mentioned work affect the interaction between the enzyme and its substrates. We therefore carried out a number of segment exchanges between the much less related $\alpha$ subunits from Burkholderia sp. strain LB400 and Rhodococcus globerulus P6, which share only about $64 \%$ sequence identity (Erickson \& Mondello, 1992; Asturias et al., 1995) and significantly differ in substrate specificity (McKay et al., 1997; Seeger et al., 1999; our unpublished results).

\section{METHODS}

Chemicals. PCB congeners ( $99 \%$ purity) were obtained from Lancaster Synthesis, from Promochem or from Restek.

Oligonucleotides. These were purchased from Life Technologies and are listed in Table 1.

Bacterial strains, plasmids and culture conditions. The strains used for general cloning were Escherichia coli DH1 (Hanahan, 1983), DH5 $\alpha$ and DH10B (Grant et al., 1990). E. coli BL21(DE3)[pLysS] (Studier, 1991) and Pseudomonas putida
KT2442 (Franklin et al., 1983) were used for gene expression. The recombinant expression vectors pAIA50 (Seeger et al., 1995a) and pDD372 (Hofer et al., 1993), harbouring bphA1A$2 A 3 A 4 B C$ or $b p h B C$ of Burkholderia sp. strain LB400 (Bopp, 1986) respectively; and pDM10 (McKay et al., 1997), harbouring bphA1A2A3A4 of R. globerulus P6 (Furukawa et al., 1978; Asturias et al., 1994), have been described previously. Bacteria were routinely grown in liquid or on solid LB medium (Sambrook et al., 1989). For strain BL21(DE3)[pLysS], the medium additionally contained chloramphenicol $\left(50 \mu \mathrm{g} \mathrm{ml}^{-1}\right)$. Ampicillin $\left(100 \mu \mathrm{g} \mathrm{ml}^{-1}\right)$ was used to select for pAIA plasmids and for pDD372. Chloramphenicol $\left(50 \mu \mathrm{g} \mathrm{ml}^{-1}\right)$ was used to select for pDM10. E. coli strains were grown at $37^{\circ} \mathrm{C}$, unless otherwise indicated. P. putida KT2442 was grown at $30^{\circ} \mathrm{C}$. Growth in liquid culture was monitored by measuring $\mathrm{OD}_{600}$.

Construction of chimaeric genes. Hybrid genes were obtained by recombinant DNA techniques which were carried out as described previously (Sambrook et al., 1989; Hofer et al., 1994), unless otherwise indicated. Hybrids bphA1-H01 and -H02 were constructed by replacement in pAIA50 of the $b p h A 1$ $M l u \mathrm{I}-A g e \mathrm{I}$ or MluI-AscI fragment of strain LB400 by the respective fragment of the $b p h A 1$ gene from strain P6. $b p h A 1$ P6 fragments were synthesized by PCR (Saiki et al., 1988). Appropriate primer pairs (LP1/LP2 or LP1/LP4, respectively) introduced the required restriction sites at their ends. The resulting plasmids were designated pAIA501 and pAIA502. $b p h A 1-\mathrm{H} 04$ was constructed as $b p h A 1-\mathrm{H} 02$, with the modification that the 3 '-terminal PCR primer (LP10) introduced a stop codon (TGA) behind codon 452 of $b p h A 1-\mathrm{P} 6$. This yielded pAIA504. $b p h A 1-\mathrm{H} 03$ was constructed like $b p h A 1-$ $\mathrm{H} 01$, with the modification that the newly inserted fragment was an LB400/P6 hybrid. This was obtained by separate PCR syntheses of the respective P6 and LB400 fragments, their purification by agarose gel electrophoresis and fusion by overlap extension (OE) PCR (Higuchi et al., 1988). The first PCR product was derived from pAIA502, using primers LP5 and LP6; the second PCR product was derived from pAIA50, using primers LP7 and LP8; both PCR products were joined 
Table 1. Oligonucleotides used

Restriction sites are underlined.

\begin{tabular}{|c|c|c|}
\hline Name & Sequence $\left(5^{\prime}-3^{\prime}\right)$ & $\begin{array}{l}\text { Restriction } \\
\text { site }\end{array}$ \\
\hline LP1 & CGCTCAAGGCACGCGTGGAGACCTACA & $M l u \mathrm{I}$ \\
\hline LP2 & CAGTCCGGGTCACCGGTGTTGGTC & AgeI \\
\hline LP4 & CCTTTCGCAGGGCGCGCCCGAATCCGGC & AscI \\
\hline LP5 & CGCTCCAGGCACGCGTGGAGA & \\
\hline LP6 & CCTTGATCTCGGCTGGAGCATCGGC & \\
\hline LP7 & GCCGATGCTCCAGCCGAGATCAAGGAAGAATATCG & \\
\hline LP8 & GACGGAACCTCGGCGCGCCGT & AscI \\
\hline LP10 & CGGCGGGCGCGCCTCAGCGGGTGGCGTTGAGGGCAGCCCAG & AscI \\
\hline LP11 & CGCTCCAGGCACGCGTGGC & $M l u \mathrm{I}$ \\
\hline LP13 & TCATTCCTGCCCACCTTCAACAACATCCGGATCTGGCACCCTCGAGGTCCCAATG & Xhol \\
\hline LP14 & GAAAACCGTㄷTATGCTGACCCG & NdeI \\
\hline LP15 & GGAGACCAACACCGGTGACCCGGACTG & AgeI \\
\hline LP16 & TTGAAGGTGGGCAGGAATGAACAGGTCGGGAAGATCGTCATATGCTGGCCGAC & NdeI \\
\hline LP17 & CAACAACATCCGGATCTGGCA & \\
\hline LP18 & AATCAGGGTGACCGGTCTGC & AgeI \\
\hline LP19 & GGCGGCGTGTTTGATCAGGACGACGGCGAGAACT & \\
\hline LP20 & TCGTCCTGATCAAACACGCCGCC & \\
\hline LP21 & GGCGACTGCGGCTTTGAC & \\
\hline LP22 & TTGCACTGGATGACCCACTTCTGCATGCCG & \\
\hline LP23 & AAGTGGGTCATCCAGTGCAA & \\
\hline LP24 & AAGCGGCCCGGAACTGGATGCCGGTGGTCG & \\
\hline LP25 & CAGTTCCGGGCCGCTT & \\
\hline LP26 & TTGAAGGTGGGCAGGAATGAA & \\
\hline
\end{tabular}

using primers LP5 and LP8. Cloning of the MluI-AgeI fragment of this product yielded pAIA503. The construction of $b p h A 1-\mathrm{H} 09$ involved several steps. Firstly, the NdeI site in the vector part of pAIA50 was eliminated by linearization with NdeI, filling in of ends and religation. This yielded pAIA55. Subsequently, two mutant fragments were derived from bphA1-LB400 by PCR, using primer pairs LP11/LP16 or LP13/LP18. The first one contained the wild-type (WT) MluI site and an NdeI site at positions 2405-2410 of the LB400 bph locus (Erickson \& Mondello, 1992), introduced by silent mutations within the 3 -terminal PCR primer. The second mutant fragment contained the WT AgeI site and an XhoI site at positions 2469-2474 of the LB400 $b p h$ locus, introduced by silent mutations within the $5^{\prime}$-terminal PCR primer. These two overlapping PCR fragments were agarose gel-purified and fused by OE-PCR with primers LP11 and LP18. The resulting mutant bphA1-LB400 fragment containing NdeI and XhoI sites was used to replace the WT MluI-AgeI fragment of pAIA55 to yield pAIA6000. Replacement of its MluI-NdeI fragment by the respective fragment from $b p h A 1-\mathrm{P} 6$, obtained by PCR with primers LP1 and LP14, yielded $b p h A 1-\mathrm{H} 09$ and pAIA509, respectively. The $b p h B C$ genes of pAIA6000, -503 , -504 and -509 , respectively, were deleted by cleavage with $B s p$ EI and recircularization to yield pAIA100, -103, -104 and -109 , respectively. Hybrids $b p h A 1-\mathrm{H} 10,-\mathrm{H} 20$ and $-\mathrm{H} 30$ were constructed by modifications of pAIA100. To obtain $b p h A 1-$ H10, the AgeI-AscI fragment of pAIA100 was exchanged for the respective fragment of pAIA504, which was PCRsynthesized using primers LP15 and LP10. The upstream primer introduced an AgeI site. This yielded pAIA110. To generate $b p h A 1-\mathrm{H} 20$, firstly the required region of pAIA504 was amplified using a hybrid LB400/P6 forward primer (LP19) and a reverse primer annealing at the AscI site (LP10). This segment was fused by OE-PCR with the respective upstream segment from pAIA100 which was synthesized with primers LP17 and LP20. The derived XhoI-AscI fragment was used to replace the respective fragment of pAIA100, yielding pAIA120. To construct $b p h A 1-\mathrm{H} 30$, the required $\mathrm{P} 6$ region was amplified from pDM10 using primers LP23 and LP24. This product was fused by OE-PCR with up- and downstream pAIA100 segments which had been amplified with primers LP21 and LP22 or LP25 and LP26, respectively. The fusion product was cleaved with $M l u \mathrm{I}$ and $\mathrm{NdeI}$, and used to replace the respective fragment of pAIA100 to yield pAIA130. The correct sequence of all cloned PCR-synthesized fragments was verified by sequencing.

DNA sequencing. This was carried out as described by Hofer et al. (1994).

Visualization of bphA1 gene products. Resting cells of E. coli BL21(DE3)[pLysS] harbouring pAIA6000 or derivatives (see below) were concentrated 10 -fold and lysed by two different methods. For SDS lysis, $2 \cdot 5 \mu$ cells were mixed with an equal volume of $2 \times$ cracking buffer (Tabor \& Richardson, 1985) and heated to $95{ }^{\circ} \mathrm{C}$ for $2 \mathrm{~min}$ prior to gel electrophoresis. Alternatively, concentrated resting cells were disrupted by two passages through a French press (Aminco) at $138 \mathrm{MPa}$. A portion $(2.5 \mu \mathrm{l})$ of the homogenate was mixed with 1 vol. $2 \times$ cracking buffer and heated as above. Electrophoresis was carried out in $0.1 \%$ SDS $/ 12 \%$ polyacrylamide gels as described previously (Hofer et al., 1993). Gels were stained with Coomassie brilliant blue R250 (Sambrook et al., 1989). 

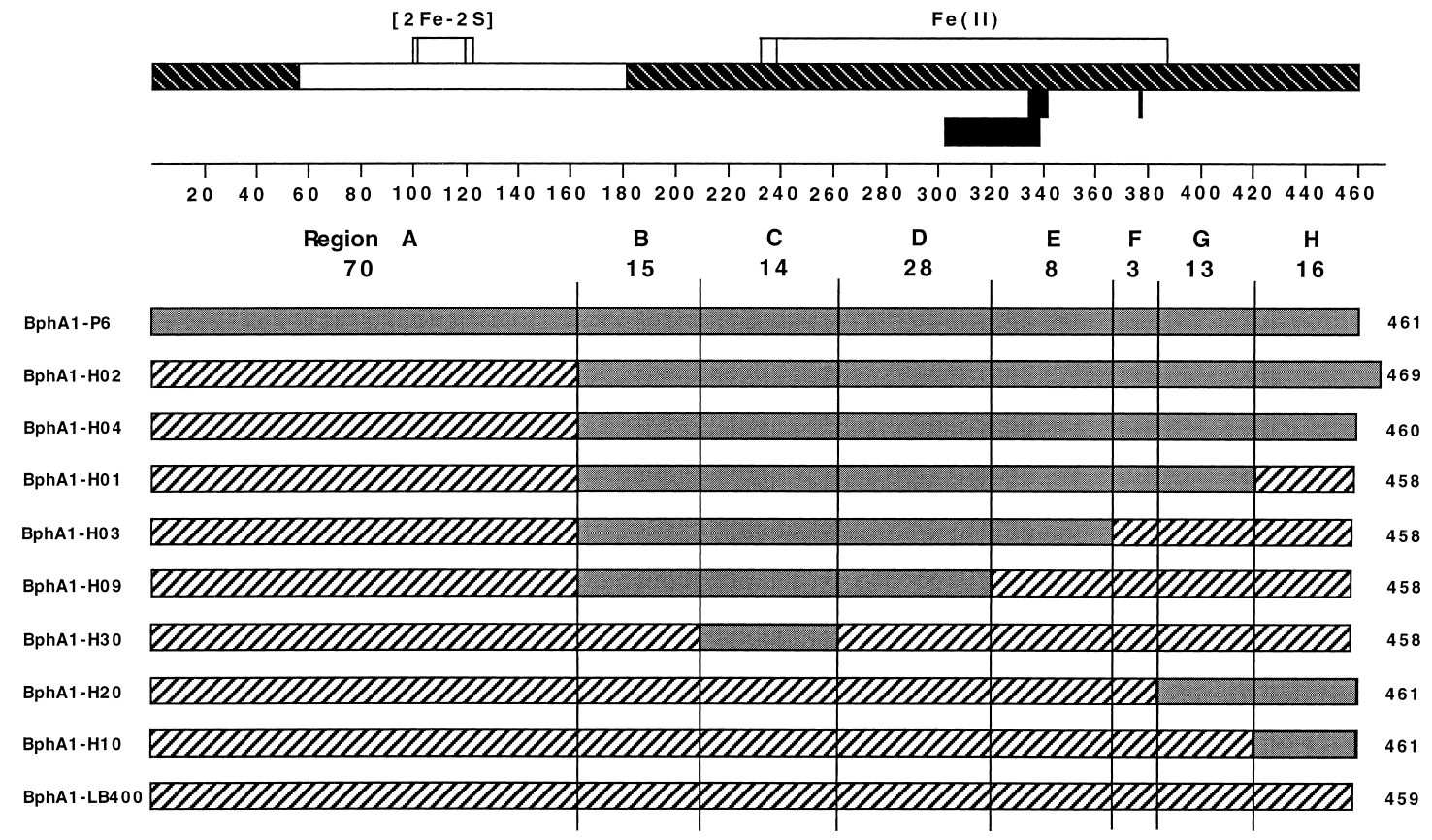

Fig. 2. Schematic representation of BphA1 WT and hybrid subunits. The $\alpha$ subunit at the top illustrates some features as deduced from the crystal structure of a naphthalene dioxygenase (Kauppi et al., 1998; Carredano et al., 2000) and from functional studies on BDO variants (Erickson \& Mondello, 1993; Kimura et al., 1997; Mondello et al., 1997). Hatched and white boxes represent the catalytic and Rieske domains, respectively. Horizontally connected vertical bars above these domains indicate the positions of amino acid ligands of the Rieske iron-sulphur cluster and of the active site mononuclear iron. Black boxes below the domains highlight regions previously reported to influence substrate specificity. Amino acid numbering is shown below. On the basis of the hybrids examined in the present work (structures shown at the bottom), the $\alpha$ subunit is divided into regions $\mathrm{A}-\mathrm{H}$. Numbers below this lettering give the number of amino acid differences (substitutions or deletions/insertions) between BphA1-LB400 and BphA1-P6. BphA1-H02 contains 11 C-terminal residues of BphA1-P6 that have no counterparts in BphA1-LB400. All other hybrids with C termini of BphA1-P6 only contain two of these amino acids. Numbers following the boxes give the total number of residues of the subunits.

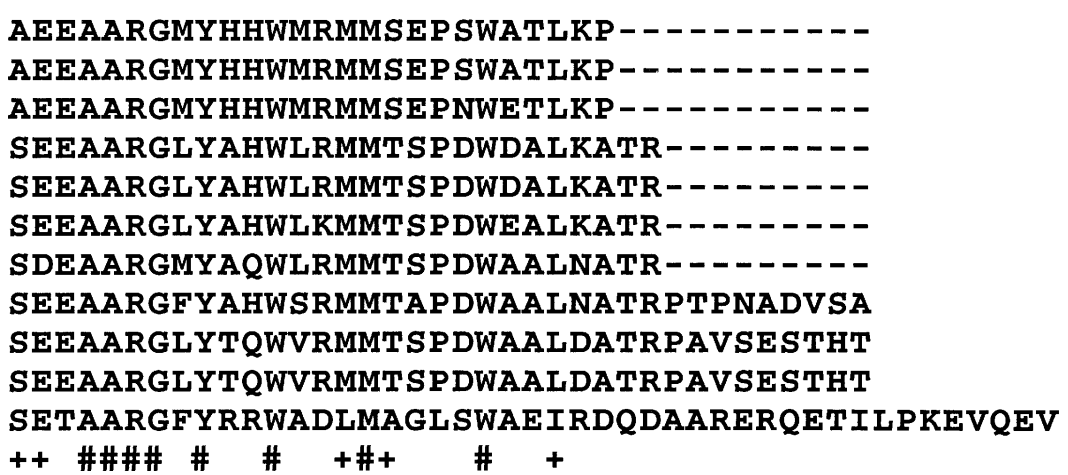
++ \#\#\# \# \# +\#+ \# +

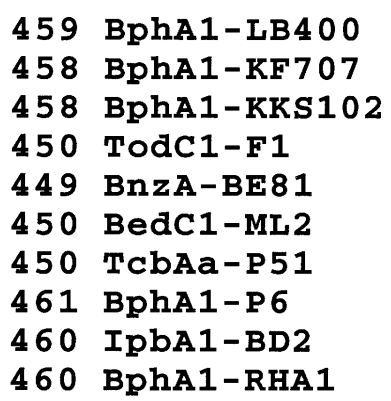

Fig. 3. Alignment of C-termini of $\alpha$ subunits of hydroxylases acting on non-condensed aromatic ring systems without polar substituents. Residues that are identical (\#) or similar $(+)$ in all proteins are indicated. The numbers of amino acid residues in the subunits are indicated. All strains are pseudomonads or related except for P6, BD2, RHA1 and TA421 which are all rhodococci. SWISS-PROT or TrEMBL primary accession numbers are: BphA1-LB400, P37333; BphA1-KF707, Q52028; BphA1-KKS102, Q52438; TodC1-F1，P13450；BnzA-BE81，P08084; BedC1-ML2, Q07944; TcbAa-P51，Q52383; BphA1-P6, Q52757; IpbA1-BD2, P72256; BphA1-RHA1, Q53122; BphA1-TA421, 069363.

Measurement of in vivo BDO activity. With cells harbouring plasmids containing $b p h A 1 A 2 A 3 A 4 B C$, BDO activity was assessed on plates by adding excess solid biphenyl to the lids and monitoring formation of 2-hydroxy-6-oxo-6-phenylhexa2,4-dienoate by eye. For quantification, resting cells of E. coli BL21(DE3)[pLysS] harbouring pAIA6000 or derivatives (see below) at a concentration of $0 \cdot 2 \mathrm{OD}_{600}$ units were incubated with biphenyl (final concentration $0 \cdot 25 \mathrm{mM}$ ) with shaking (175 r.p.m.) at $30^{\circ} \mathrm{C}$, and the formation of 2-hydroxy-6-oxo6-phenylhexa-2,4-dienoate was monitored photometrically $\left(A_{434}\right)$. With plasmids harbouring $b p h A 1 A 2 A 3 A 4$, activity was assayed likewise, with the exception that the resting cells 
(final concentration $0 \cdot 1 \mathrm{OD}_{600}$ unit) were mixed with resting cells harbouring pDD372 (final concentration $0 \cdot 2 \mathrm{OD}_{600}$ units), which directs the syntheses of 2,3-dihydro-2,3-dihydroxybiphenyl-2,3 dehydrogenase and 2,3-dihydroxybiphenyl-1,2 dioxygenase.

Preparation of resting cells. E. coli BL21(DE3)[pLysS] harbouring pAIA6000 or derivatives was grown at $37{ }^{\circ} \mathrm{C}$ in $\mathrm{LB}$ medium with antibiotics to an $\mathrm{OD}_{600}$ between $1 \cdot 1$ and $1 \cdot 3$. IPTG was added to $0.4 \mathrm{mM}$ final concentration and the incubation continued at $30^{\circ} \mathrm{C}$ for another $30 \mathrm{~min}$. Cells were harvested, washed with $50 \mathrm{mM}$ sodium phosphate buffer, $\mathrm{pH} 8 \cdot 0$, containing $5 \mathrm{mM}$ glucose and resuspended in the same buffer to a final concentration of $\mathrm{OD}_{600}=5 \cdot 0$. As WT dioxygenase activity from strain P6 could not be measured in E. coli (McKay et al., 1997; Chebrou et al., 1999), the respective genes were expressed in $P$. putida KT2442 as previously described (McKay et al., 1997). Resting cells of $P$. putida were prepared as above.

Resting cell assays. Single chlorobiphenyls (CBs) were added to $1 \mathrm{ml}$ resting cells to a final concentration of $0.75 \mathrm{mM}$. The Teflon-sealed tubes were shaken at 175 r.p.m. at $30{ }^{\circ} \mathrm{C}$ for $24 \mathrm{~h}$. Thereafter, $50 \mu \mathrm{l} 25 \mathrm{mM}$ 2,2'-dihydroxybiphenyl was added as a standard and the suspensions were extracted with 1 vol. ethylacetate by inverting several times. A portion $(10 \mu \mathrm{l})$ of the organic phase was analysed in a high performance liquid chromatograph (Shimadzu LC10AD) equipped with a diode array detector and an SC Lichrosphere 100 RP8 $5 \mu \mathrm{m}$ column (length, $125 \mathrm{~mm}$; internal diameter, $4.6 \mathrm{~mm}$ ) (Bischoff). The aqueous eluent contained $1 \mathrm{ml} 85 \%$ ortho-phosphoric acid and $600 \mathrm{ml}$ methanol $\mathrm{l}^{-1}$. The flow rate was $1 \mathrm{ml} \mathrm{min}^{-1}$. Metabolites were quantified from HPLC peak areas which had been normalized to the internal standard. Percentages of metabolites were calculated on the basis that the sum of all metabolites formed by a given enzyme represents $100 \%$. HPLC peak areas were derived from absorptions at the wavelengths indicated in Table 3. As molar extinction coefficients are unknown, molar amounts could not be determined. Thus, the given percentages of metabolites do not necessarily represent molar ratios.

\section{RESULTS}

\section{Construction of hybrid genes and proteins}

Additional restriction sites were introduced into the bphA1 gene of Burkholderia sp. strain LB400 by PCRbased site-specific mutagenesis. Subsequently, segments of the bphA1 gene of R. globerulus P6 were obtained by PCR. In most cases, restriction sites were used to directly replace the respective LB400 segments. If restriction sites were not appropriately positioned, LB400-P6 fusions were first generated by OE-PCR which subsequently could be introduced into the LB400 $b p h A$ gene cluster via restriction sites. After cloning, all segments obtained by PCR were resequenced. Errors were occasionally found in these regions. An overview of the segments exchanged in the different constructs is shown in Fig. 2.

Growth problems were repeatedly observed with the E. coli strain synthesizing BphA1-H02, which was one of the earliest hybrids constructed. A sequence alignment indicated that $\alpha$ subunits of ARHDOs attacking hydrophobic benzene derivatives with non-condensed rings, such as biphenyls, appear to possess stretches of extra Cterminal amino acids only if they were produced by

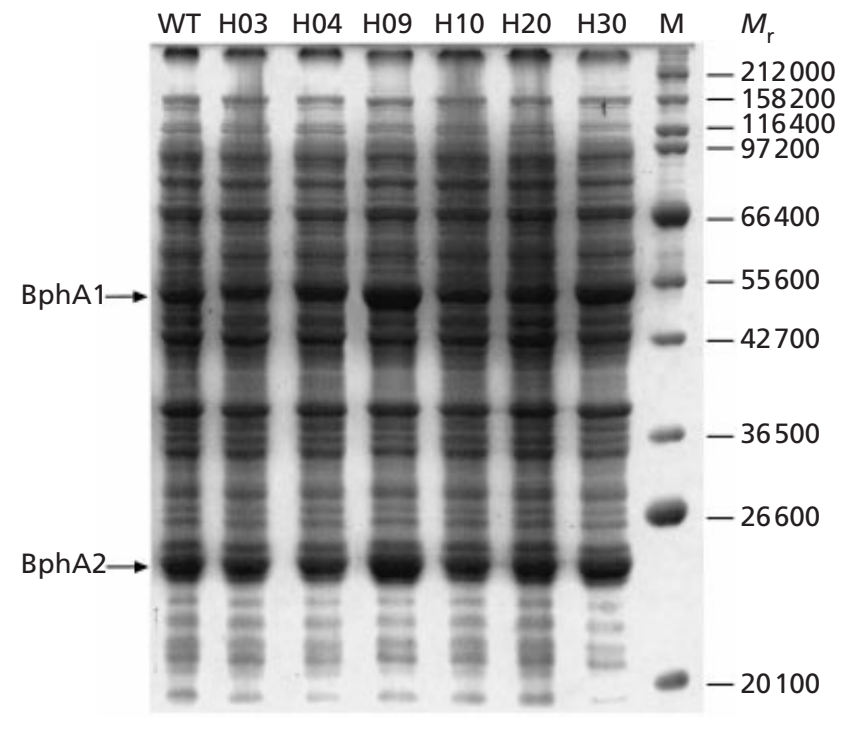

Fig. 4. SDS-PAGE of BphA1 and BphA2 subunits. E. coli $B$ 21(DE3)[pLysS] was used as the host strain. The BDO type is given above the lanes.

Table 2. Activity of BDOs in recombinant cells of $E$. coli BL21(DE3)[pLysS]

$\mathrm{m} A_{434}$ refers to the concentration of 2-hydroxy-6-oxo-6phenylhexa-2,4-dienoate, determined as $A_{434}$. $\mathrm{OD}_{600}$ refers to the concentration of cells. Standard deviations were $10-30 \%$.

\begin{tabular}{|cc|}
\hline BDO & $\begin{array}{c}\text { Activity in vivo } \\
{\left[\mathbf{m} A_{434} /\left(\mathrm{OD}_{\mathbf{6 0 0}} \times \mathbf{m i n}\right)\right]}\end{array}$ \\
\hline LB400 & 65 \\
H01 & No activity \\
H02 & Not assayed \\
H03 & 20 \\
H04 & $7 \cdot 4$ \\
H09 & $8 \cdot 5$ \\
H10 & 93 \\
H20 & $4 \cdot 0$ \\
H30 & 20 \\
\hline
\end{tabular}

Gram-positive organisms (Fig. 3). We therefore constructed $b p h A 1-\mathrm{H} 04$ which does not encode these amino acids, but is otherwise identical to bphA1-H02. This eliminated the growth problems and led to the synthesis of an active subunit (see below). Therefore all other hybrids containing the C-terminus from strain P6 were constructed without these nine residues.

\section{Cellular concentration of WT and hybrid BphA1 subunits and activity of the resulting BDOs}

Using E. coli BL21(DE3)[pLysS] as host strain, the concentrations of WT and hybrid BphA1 subunits were monitored after SDS-PAGE separation of total cellular proteins obtained by treatment of cells with cracking 

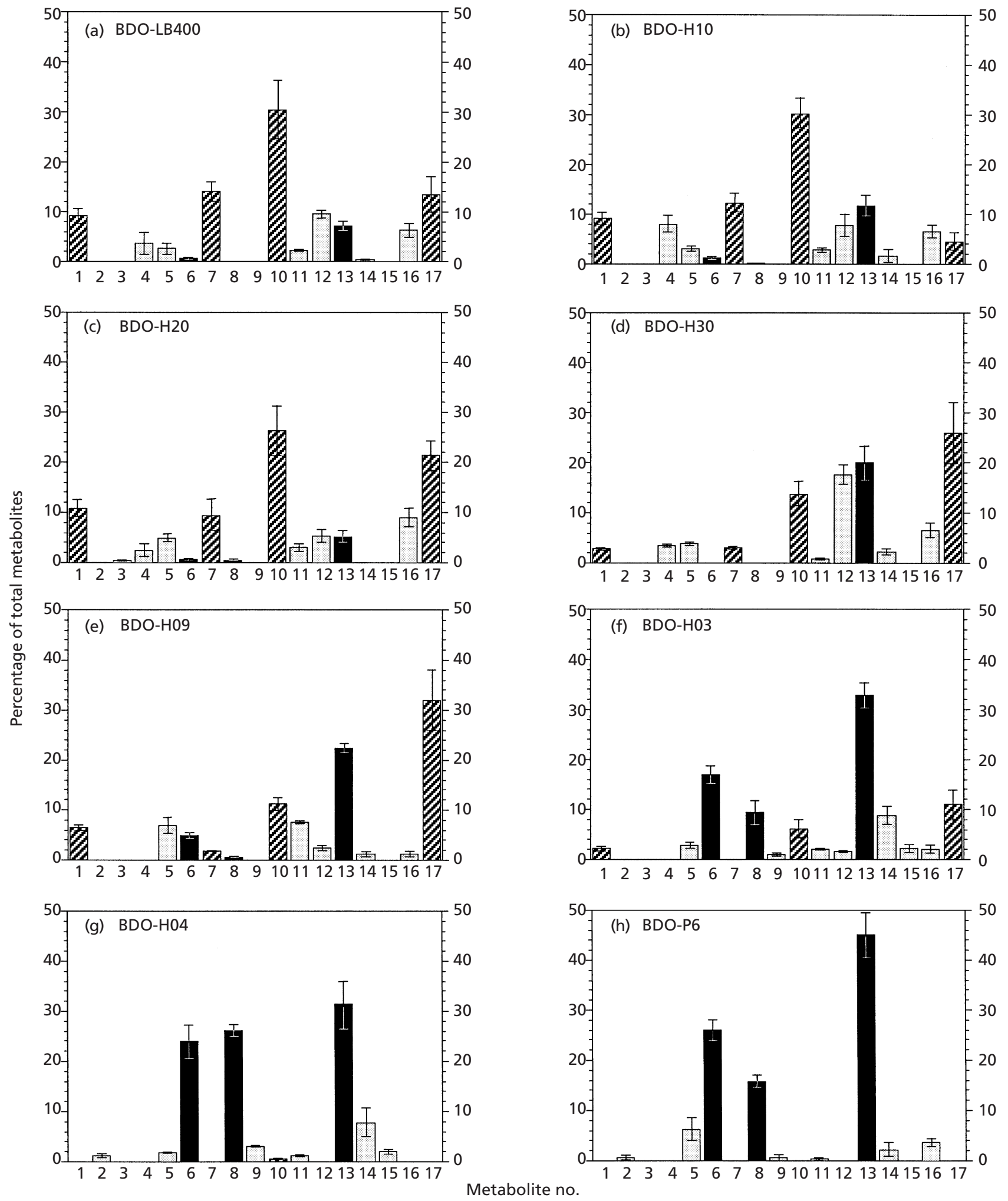

Fig. 5. Product spectra of wild-type and hybrid dioxygenases with selected CBs as substrates. Types of dioxygenases are indicated inside each diagram. Percentages of metabolites as derived from HPLC peak areas (for details see Methods) are indicated. The sum of all metabolites formed by a given enzyme represents $100 \%$. Columns depicting metabolites that are particularly prominent with BDO-LB400 or -P6 are hatched or solid, respectively. Columns representing other metabolites are shaded grey. Standard deviations are indicated. For substrates and characteristics of metabolites, refer to Table 3. 
Table 3. Characteristics of CB metabolites

\begin{tabular}{|c|c|c|c|c|c|}
\hline \multirow[t]{3}{*}{ Substrate } & \multicolumn{5}{|c|}{ Product } \\
\hline & \multirow[t]{2}{*}{ No. } & \multirow[t]{2}{*}{$\mathrm{t}_{\mathrm{r}}(\min )$} & \multirow[t]{2}{*}{$\lambda_{\max }(\mathrm{nm})^{*}$} & \multicolumn{2}{|r|}{ Site of dioxygenation } \\
\hline & & & & Assignment & Reference \\
\hline \multirow[t]{3}{*}{$2,2^{\prime}-\mathrm{CB}$} & 1 & $5 \cdot 5$ & $201, \underline{279}$ & 2,3 & Haddock et al. (1995b); Seeger et al. (1995b) \\
\hline & 2 & $5 \cdot 6$ & $206, \underline{276}$ & $5,6+$ & \\
\hline & 3 & $7 \cdot 5$ & $202, \underline{285}$ & 3,4 or $4,5+$ & \\
\hline \multirow[t]{4}{*}{$2,3^{\prime}-\mathrm{CB}$} & 4 & $3 \cdot 6$ & $208, \underline{255}$ & $4^{\prime}, 5^{\prime} \dagger$ & \\
\hline & 5 & $7 \cdot 4$ & $209,232(\mathrm{~s}), \underline{280}$ & $5,6 \dagger$ & \\
\hline & 6 & $7 \cdot 9$ & $202, \underline{285}$ & $5^{\prime}, 6^{\prime}$ & $\begin{array}{l}\text { M. Seeger, M. Zielinski and B. Hofer, } \\
\text { unpublished results }\end{array}$ \\
\hline & 7 & $9 \cdot 4$ & $206, \underline{250}$ & 2,3 & Haddock et al. (1995b) \\
\hline \multirow[t]{3}{*}{$2,4^{\prime}-\mathrm{CB}$} & 8 & $5 \cdot 5$ & $203, \underline{290}$ & $2^{\prime}, 3^{\prime}$ & $\begin{array}{l}\text { M. Seeger, M. Zielinski and B. Hofer, } \\
\text { unpublished results }\end{array}$ \\
\hline & 9 & $8 \cdot 1$ & $<200,288, \underline{227}$ & $5,6+$ & \\
\hline & 10 & $9 \cdot 4$ & $201, \underline{253}$ & 2,3 & Seeger et al. (1995b) \\
\hline \multirow[t]{3}{*}{$3,4^{\prime}-\mathrm{CB}$} & 11 & $6 \cdot 2$ & $\underline{313}, \overline{205}$ & $4,5 \dagger$ & \\
\hline & 12 & $8 \cdot 0$ & $\underline{244}, 202,288$ & $2^{\prime}, 3^{\prime} \dagger$ & \\
\hline & 13 & $8 \cdot 9$ & $<200, \underline{309}, 231$ & 5,6 & McKay et al. (1997) \\
\hline $4,4^{\prime}-\mathrm{CB}$ & 14 & $6 \cdot 2$ & $<200, \underline{316}, 232$ & 2,3 & Seeger et al. (1995a); McKay et al. (1997) \\
\hline \multirow[t]{2}{*}{$2,5,4^{\prime}-\mathrm{CB}$} & 15 & $9 \cdot 0$ & $<200, \overline{220}(\mathrm{~s}), \underline{290}$ & $2^{\prime}, 3^{\prime} \dagger$ & \\
\hline & 16 & $10 \cdot 1$ & $244,205,293$ & $3,4 \dagger$ & \\
\hline $2,5,2^{\prime}, 5^{\prime}-\mathrm{CB}$ & 17 & $13 \cdot 8$ & $\overline{<200,220,} 288$ & 3,4 & Haddock et al. (1995b) \\
\hline
\end{tabular}

* Maxima are listed in order of decreasing absorption. Wavelengths used for metabolite quantification are underlined. s, shoulder. † Tentative assignment.

buffer or by disruption of cells with a French press. Both methods yielded identical results. All $\alpha$ subunits were present in comparable amounts that could be visualized by conventional staining with Coomassie blue (Fig. 4). This includes subunit BphA1-H01 (not shown in Fig. 4) which did not yield an active BDO (below). The catalytic activity of the respective hybrid BDO systems was assayed in vivo with resting cells of E. coli BL21(DE3) [pLysS] harbouring pAIA6000 or derivatives and with biphenyl as substrate. All enzymes except for BDO-H01 were active. Activities varied up to 30-fold (Table 2).

\section{Discrimination between regions of major and minor influence on the topology of substrate binding}

A change in the regiospecificity of dioxygenation of a given substrate reflects a change in the orientation of the substrate relative to the position of the activated dioxygen at the BDO active site and/or a change in the electron density at substrate carbons. Thus it is a sensitive measure for changes in the steric-electronic structure of the substrate-binding pocket. As no single compound is able to sense all possible structural alterations, seven $\mathrm{CBs}$ were used as probes. They were chosen based on the criterion that they yielded significantly different product profiles when dioxygenated by the two parental BDOs used to construct the chimaeras (McKay et al., 1997; Seeger et al., 1999; our unpublished results).
The distributions of products obtained with WT and chimaeric enzymes are shown in Fig. 5. Analytical data of the metabolites are given in Table 3. In Fig. 5, the columns representing marker metabolites that are particularly prominent with BDO-LB400 or -P6 are shown hatched or solid, respectively. Correlation with Table 3 shows that the four marker metabolites for BDO-LB400 reflect dehalogenating attacks at carbons 2 and 3 of $2,2^{\prime}-, 2,3^{\prime}-$ or $2,4^{\prime}-\mathrm{CB}$, respectively, and the meta,paradioxygenation of $2,5,2^{\prime}, 5^{\prime}$-CB. The three marker metabolites for BDO-P6 were formed by non-dechlorinating ortho,meta-dioxygenation of the meta-chlorinated ring of 2,3' - and 3,4'-CB, and of the para-chlorinated ring of $2,4^{\prime}-\mathrm{CB}$.

As shown in Fig. 5, BDO-H10 and BDO-H20 produced product patterns quite similar to that of BDO-LB400. BDO-H09 and BDO-H30, in which a subsegment of the former was exchanged, shared a certain degree of similarity with each other and behaved clearly differently from both parental BDOs. Particularly the positioning of the substrate leading to dehalogenating dioxygenation was reduced with all three of the respective substrates, whilst the attack in position 3 and 4 of $2,5,2^{\prime}, 5^{\prime}$-CB was maintained. The 5,6-dioxygenation of the meta-substituted ring of 3,4'-CB was significantly enhanced. BDO-H03 showed increased similarity with BDO-P6. The amounts of the P6 marker metabolites derived from 2,3'- and 2,4'-CB were clearly enhanced. Most of the LB400 marker metabolites, including 
3,4-dihydroxy-2,5,2',5'-tetrachlorobiphenyl, were still formed, albeit at significantly reduced amounts. BDOH04 behaved very similarly to BDO-P6, although the $\mathrm{N}$-terminal 165 aa of the $\alpha$ subunit and all other BDO subunits were derived from the LB400 enzyme.

\section{DISCUSSION}

Hybrid dioxygenase systems were generated to discriminate between enzyme segments of major and minor importance for the structure of the substrate-binding pocket. They consisted of WT proteins BphA2, -A3 and -A4 from strain LB400 and of chimaeric BphA1 or $\alpha$ subunits. Whilst BphA1 and BphA2 form a heterohexamer (Haddock et al., 1995a) that carries out substrate hydroxylation, BphA3 and BphA4 transport electrons to this hydroxylase complex and are therefore less likely to affect enzyme-substrate interaction.

The hybrid dioxygenase BDO-H04 possessed substrate and product spectra that closely resembled those of the enzyme from $R$. globerulus P6. This result, in view of the $43 \%$ sequence difference between BphA2-LB400 and $-\mathrm{P} 6$, suggests that the $\beta$ subunits exert no major influence on the structure of the active site. We note that these findings contrast those of Hurtubise et al. (1998) and Chebrou et al. (1999), who reported a significant contribution of the $\beta$ subunit to BDO-substrate interaction. However, a recent publication from this group (Barriault et al., 2001) reported no major influence of the small subunit on substrate specificity. Based on all fusions generated, the $\alpha$ subunit may be divided into eight segments, designated $\mathrm{A}$ to $\mathrm{H}$ (Fig. 2). The $\mathrm{N}$-terminal region $\alpha \mathrm{A}$ harbours the Rieske iron-sulphur cluster (Fig. 2). In BDO-H04, $\alpha \mathrm{A}$ was derived from BDO-LB400. The corresponding region of BDO-P6 differs in $70(42 \%)$ of the residues. Thus, the highly similar regiospecificity of dioxygenation by BDO-H04 and BDO-P6 makes it unlikely that region $\alpha \mathrm{A}$ significantly participates in formation of the substrate-binding pocket, although an $\alpha$ subunit sequence alignment with a naphthalene dioxygenase, for which three-dimensional crystal structures have been determined (Kauppi et al., 1998; Carredano et al., 2000), suggests that the Nterminal residues up to about position 55 are part of the 'catalytic domain' (Fig. 2) that harbours the active site. Barriault et al. (2001) exchanged the $\alpha \mathrm{A}$ regions between the BDOs from Comamonas testosteroni B-356 and strain LB400, which differ at 28 positions. Whilst the B-356 variant containing $\alpha$ A from strain LB400 behaved very similarly to the B-356 WT, the reverse replacement showed significant differences in turnover of two of the four CBs assayed. This suggests a context effect on these exchanges (see below).

Chimaeras BDO-H10 and -H20 examined the other end of the $\alpha$ subunit. Their product profiles were almost identical to that of BDO-LB400. The exchanged regions at the $\mathrm{C}$ terminus differ by almost $40 \%$ from the LB400 sequence. This indicates that the C-terminal 75 aa (regions $\alpha \mathrm{G}$ and $\alpha \mathrm{H}$ ) exert an almost negligible effect on substrate dioxygenation.
Apparently, in BDO-H03, -H09 and -H30, the structure of the active site was influenced by peptide segments from both sources. The conversion of $2,4^{\prime}-\mathrm{CB}$ indicated a significant difference between BDO-H03 and -H04. As regions $\alpha \mathrm{G}$ and $\alpha \mathrm{H}$ have been shown to be of minor importance, this result highlights a significant effect of region $\alpha$ F. Previously, Kimura et al. (1997) and Mondello et al. (1997) identified a major contribution of aa 377 (LB400 numbering) on substrate specificity. This is one of the three amino acid differences between regions $\alpha \mathrm{F}$ of the LB400 and P6 subunits.

Differences in CB dioxygenation between BDO-H09 and -H03 clearly demonstrate the importance of region $\alpha \mathrm{E}$, in agreement with findings made in the LB400/KF707 system (Erickson \& Mondello, 1993; Kimura et al., 1997; Mondello et al., 1997). However, the difference in CB dioxygenation between BDO-H09 and BDO-LB400 was not expected on the basis of those studies. It revealed that sequence elements $\mathrm{N}$-terminal to position 320 significantly influence the substrate-binding site. A first indication for this was provided by Kumamaru et al. (1998) who found that a random BDO variant which had obtained substitutions of aa 255, 258 and 303 showed an altered substrate specificity. The segment exchanged in BDO-H09, by analogy with other ARHDOs, is likely to comprise amino acid ligands of the active site mononuclear iron (Fig. 2) (Jiang et al., 1996; Butler \& Mason, 1997; Kauppi et al., 1998). We therefore constructed a hybrid, BDO-H30, in which primarily these sequence elements were replaced. Also this variant clearly behaved differently from BDOLB400, demonstrating that exchange of this subregion indeed altered enzyme-substrate interactions. Although investigations of different ARHDOs are of limited comparability, we note that Beil et al. (1998) and Parales et al. (2000) reported effects of amino acid substitutions at five or two positions, respectively, within the corresponding regions of a toluene or a naphthalene dioxygenase, respectively. Barriault et al. (2001), however, exchanging a region between BDOs of strains LB400 and B-356 that comprises the region substituted in BDO-H30, found only a minor influence on substrate specificity. These differences in results may be a consequence of the different assays used. They may also be due to the fact that amino acid replacements in these experiments (partly) differ in positions, in types of substitutions and in flanking sequences ('context dependence'). If only a fraction of the amino acid residues that determine a given property are exchanged, context dependence of the effects of such replacements is inevitable. This and other publications (Beil et al., 1998; Kauppi et al., 1998; Carredano et al., 2000; Parales et al., 2000) suggest that the structure of the substrate-binding site is determined by segments that are rather distant in the sequence. Thus, context dependence is expected for the effects of individual exchanges of such segments on the structure of the binding site. Therefore, generalizations in the interpretation of experiments must be made with caution.

Problems were encountered in the biosynthesis of the 
BDO from R. globerulus $\mathrm{P} 6$ in recipient organisms. Whilst active BDO-P6 was obtained in P. putida cells grown under specific conditions, neither activity nor BDO proteins were detected in E. coli (McKay et al., 1997). Numerous examples indicate that insufficient initiation of translation is a major obstacle in the expression of cloned genes. Inefficient initiation in most cases is related to the new combination of $5^{\prime}$-untranslated and/or -translated gene regions. Recently, Chebrou et al. (1999) successfully used a different expression vector additionally encoding a $5^{\prime}$-terminal His-tag to achieve synthesis of the $\mathrm{BDO} \alpha$ and $\beta$ subunits from strain P6 in E. coli. However, these authors encountered another problem. The hydroxylase formed was catalytically inactive due to incorrect assembly of the iron-sulphur cluster. Results of the present study indicate that both difficulties may be circumvented by one and the same approach. The generation of P6-like BDO activity in E. coli by a hybrid enzyme exemplifies that fusion of the 'problematic' gene with a 'helper' cistron is a feasible strategy to obtain a specific ARHDO activity in a host organism that is inappropriate to carry out synthesis of the respective WT enzyme in an active form.

\section{ACKNOWLEDGEMENTS}

The authors thank Annette Krüger and Ed Moore for DNA sequencing support and gratefully acknowledge funding of this work through a grant from the Deutsche Forschungsgemeinschaft (Ho 1219/2-1).

\section{REFERENCES}

Asturias, J. A., Moore, E., Yakimov, M. M., Klatte, S. \& Timmis, K. N. (1994). Reclassification of the polychlorinated biphenyldegraders Acinetobacter sp. strain P6 and Corynebacterium sp. strain MB1 as Rhodococcus globerulus. Syst Appl Microbiol 17, 226-231.

Asturias, J. A., Diaz, E. \& Timmis, K. N. (1995). The evolutionary relationship of biphenyl dioxygenase from Gram-positive Rhodococcus globerulus $\mathrm{P} 6$ to multicomponent dioxygenases from Gram-negative bacteria. Gene 156, 11-18.

Barriault, D., Simard, C., Chatel, H. \& Sylvestre, M. (2001). Characterization of hybrid biphenyl dioxygenases obtained by recombining Burkholderia sp. strain LB400 bphA with the homologous gene of Comamonas testosteroni B-356. Can J Microbiol 47, 1025-1032.

Beil, S., Mason, J. R., Timmis, K. N. \& Pieper, D. H. (1998). Identification of chlorobenzene dioxygenase sequence elements involved in dechlorination of 1,2,4,5-tetrachlorobenzene. J Bacteriol 180, 5520-5528.

Bopp, L. H. (1986). Degradation of highly chlorinated PCBs by Pseudomonas strain LB400. J Ind Microbiol 1, 23-29.

Boyd, D. R. \& Sheldrake, G. N. (1998). The dioxygenase-catalyzed formation of vicinal cis-diols. Nat Prod Rep 15, 309-325.

Butler, C. S. \& Mason, J. R. (1997). Structure-function analysis of the bacterial aromatic ring-hydroxylating dioxygenases. $A d v$ Microb Physiol 38, 47-84.

Carredano, E., Karlsson, A., Kauppi, B. \& 7 other authors (2000). Substrate binding site of naphthalene 1,2-dioxygenase: functional implications of indole binding. J Mol Biol 296, 701-712.
Chebrou, H., Hurtubise, Y., Barriault, D. \& Sylvestre, M. (1999). Heterologous expression and characterization of the purified oxygenase component of Rhodococcus globerulus P6 biphenyl dioxygenase and of chimeras derived from it. J Bacteriol 181, 4805-4811.

Erickson, B. D. \& Mondello, F. (1992). Nucleotide sequencing and transcriptional mapping of the genes encoding biphenyl dioxygenase, a multicomponent polychlorinated-biphenyl-degrading enzyme in Pseudomonas strain LB400. J Bacteriol 174, 2903-2912.

Erickson, B. D. \& Mondello, F. J. (1993). Enhanced biodegradation of polychlorinated biphenyls after site-directed mutagenesis of a biphenyl dioxygenase gene. Appl Environ Microbiol 59, 38583862.

Franklin, F. C. H., Bagdasarian, M., Bagdasarian, M. M. \& Timmis, K. N. (1983). Molecular and functional analysis of the TOL plasmid pWWO from Pseudomonas putida and cloning of genes for the entire regulated aromatic ring meta cleavage pathway. Proc Natl Acad Sci US A 78, 7458-7462.

Furukawa, K., Matsumura, F. \& Tonomura, K. (1978). Alcaligenes and Acinetobacter strains capable of degrading polychlorinated biphenyls. Agric Biol Chem 42, 543-548.

Furukawa, K., Hirose, J., Hayashida, S. \& Nakamura, K. (1994). Efficient degradation of trichloroethylene by a hybrid aromatic ring dioxygenase. J Bacteriol 176, 2121-2123.

Gibson, D. T. \& Parales, R. E. (2000). Aromatic hydrocarbon dioxygenases in environmental biotechnology. Curr Opin Biotechnol 11, 236-243.

Grant, S. G. N., Jesse, J., Bloom, F. R. \& Hanahan, D. (1990). Differential plasmid rescue from transgenic mouse DNAs into Escherichia coli methylation-restriction mutants. Proc Natl Acad Sci U S A 87, 4645-4649.

Haddock, J. D., Horton, J. R. \& Gibson, D. T. (1995a). Purification and characterization of the oxygenase component of biphenyl 2,3-dioxygenase from Pseudomonas sp. strain LB400. J Bacteriol 177, 5834-5839.

Haddock, J. D., Horton, J. R. \& Gibson, D. T. (1995b). Dihydroxylation and dechlorination of chlorinated biphenyls by purified biphenyl 2,3-dioxygenase from Pseudomonas sp. strain LB400. J Bacteriol 177, 20-26.

Hanahan, D. (1983). Studies on transformation of Escherichia coli with plasmids. J Mol Biol 166, 557-580.

Higuchi, R., Krummel, B. \& Saiki, R. K. (1988). A general method of in vitro preparation and specific mutagenesis of DNA fragments: study of protein and DNA interactions. Nucleic Acids Res 16, 7351-7367.

Hirose, J., Suyama, A., Hayashida, S. \& Furukawa, K. (1994). Construction of hybrid biphenyl $(b p h)$ and toluene $(t o d)$ genes for functional analysis of aromatic ring dioxygenases. Gene 138, 27-33.

Hofer, B., Eltis, L. D., Dowling, D. N. \& Timmis, K. N. (1993). Genetic analysis of a Pseudomonas locus encoding a pathway for biphenyl/polychlorinated biphenyl (PCB) degradation. Gene 130, 47-55.

Hofer, B., Backhaus, S. \& Timmis, K. N. (1994). The biphenyl/ polychlorinated biphenyl-degradation locus $(b p h)$ of Pseudomonas sp. LB400 encodes four additional metabolic enzymes. Gene 144, 9-16.

Hudlicky, T., Gonzalez, D. \& Gibson, D. T. (1999). Enzymatic hydroxylation of aromatics in enantioselective synthesis: expanding asymmetric methodology. Aldrichim Acta 32, 35-62.

Hurtubise, Y., Barriault, D. \& Sylvestre, M. (1998). Involvement of the terminal oxygenase beta subunit in the biphenyl dioxygenase 
reactivity pattern toward chlorobiphenyls. J Bacteriol 180, 5828-5835.

Jiang, H., Parales, R. E., Lynch, N. A. \& Gibson, D. T. (1996). Sitedirected mutagenesis of conserved amino acids in the alpha subunit of toluene dioxygenase: potential mononuclear nonheme iron coordination sites. J Bacteriol 178, 3133-3139.

Kauppi, B., Lee, K., Carredano, E., Parales, R. E., Gibson, D. T., Eklund, H. \& Ramaswamy, S. (1998). Structure of an aromaticring-hydroxylating dioxygenase - naphthalene 1,2-dioxygenase. Structure 6, 571-586.

Kimura, N., Nishi, A., Goto, M. \& Furukawa, K. (1997). Functional analyses of a variety of chimeric dioxygenases constructed from two biphenyl dioxygenases that are similar structurally but different functionally. J Bacteriol 179, 3936-3943.

Kumamaru, T., Suenaga, H., Mitsuoka, M., Watanabe, T. \& Furukawa, K. (1998). Enhanced degradation of polychlorinated biphenyls by directed evolution of biphenyl dioxygenase. Nat Biotechnol 16, 663-666.

McKay, D. B., Seeger, M., Zielinski, M., Hofer, B. \& Timmis, K. N. (1997). Heterologous expression of biphenyl dioxygenase-encoding genes from a Gram-positive broad-spectrum PCB degrader and characterization of chlorobiphenyl oxidation by the gene products. J Bacteriol 179, 1924-1930.

Mondello, F. J., Turcich, M. P., Lobos, J. H. \& Erickson, B. D. (1997). Identification and modification of biphenyl dioxygenase sequences that determine the specificity of polychlorinated biphenyl degradation. Appl Environ Microbiol 63, 3096-3103.

Parales, R. E., Lee, K., Resnick, S. M., Jiang, H., Lessner, D. J. \& Gibson, D. T. (2000). Substrate specificity of naphthalene dioxygenase: effect of specific amino acids at the active site of the enzyme. J Bacteriol 182, 1641-1649.
Saiki, R. K., Gelfand, D. H., Stoffel, S., Scharf, S. J., Higuchi, R., Horn, G. T., Mullis, K. B. \& Erlich, H. A. (1988). Primer-directed enzymatic amplification of DNA with a thermostable DNA polymerase. Science 239, 487-491.

Sambrook, J., Fritsch, E. F. \& Maniatis, T. (1989). Molecular Cloning: a Laboratory Manual, 2nd edn, Cold Spring Harbor NY: Cold Spring Harbor Laboratory.

Seeger, M., Timmis, K. N. \& Hofer, B. (1995a). Conversion of chlorobiphenyls into phenylhexadienoates and benzoates by the enzymes of the upper pathway for polychlorobiphenyl degradation encoded by the $b p h$ locus of Pseudomonas sp. strain LB400. Appl Environ Microbiol 61, 2654-2658.

Seeger, M., Timmis, K. N. \& Hofer, B. (1995b). Degradation of chlorobiphenyls catalyzed by the $b p h$-encoded biphenyl-2,3dioxygenase and biphenyl-2,3-dihydrodiol-2,3-dehydrogenase of Pseudomonas sp. LB400. FEMS Microbiol Lett 133, 259-264.

Seeger, M., Zielinski, M., Timmis, K. N. \& Hofer, B. (1999). Regiospecificity of dioxygenation of di- to pentachlorobiphenyls and their degradation to chlorobenzoates by the $b p h$-encoded catabolic pathway of Burkholderia sp. strain LB400. Appl Environ Microbiol 65, 3614-3621.

Studier, F. W. (1991). Use of bacteriophage T7 lysozyme to improve an inducible T7 expression system. J Mol Biol 219, $37-44$.

Tabor, S. \& Richardson, C. C. (1985). A bacteriophage T7 RNA polymerase/promoter system for controlled exclusive expression of specific genes. Proc Natl Acad Sci U S A 82, 1074-1078.

Received 31 December 2001; revised 29 April 2002; accepted 7 May 2002. 